\title{
Efficacy analysis of endoscopic submucosal dissection in gastric adenocarcinoma of fundic gland type: five cases of more than 36 months of follow-up
}

\author{
Yang Li", Youzhu Lu\#, Chao Yu, Jun Xiao, Shutang Han \\ Gastroenterology Endoscopy Center, Affiliated Hospital of Nanjing University of Chinese Medicine (Jiangsu Province Hospital of Chinese \\ Medicine), Nanjing 210000, China \\ Contributions: (I) Conception and design: Y Li, S Han; (II) Administrative support: Y Li, Y Lu, J Xiao, S Han; (III) Provision of study materials or \\ patients: All authors; (IV) Collection and assembly of data: All authors; (V) Data analysis and interpretation: Y Li, Y Lu, S Han; (VI) Manuscript \\ writing: All authors; (VII) Final approval of manuscript: All authors. \\ \#These authors contributed equally to this work. \\ Correspondence to: Shutang Han, MD, PhD. Gastroenterology Endoscopy Center, Affiliated Hospital of Nanjing University of Chinese Medicine, \\ Nanjing 210000, China. Email: shutanghanhst@163.com.
}

\begin{abstract}
Background: To explore the feasibility, safety, and effectiveness of endoscopic submucosal dissection (ESD) in the treatment of gastric adenocarcinoma of fundic gland type (GA-FG).

Methods: A retrospective analysis was performed for lesion characteristics, treatment, and follow-up of 5 cases of GA-FG patients treated with ESD in our endoscopy center from Jan 2010 to Dec 2018.

Results: Among the 5 patients, there were 2 males and 3 females ( $M: F=2: 3$ ). The average age was $59.8 \pm 14.4$ years old. Four lesions were located at the fundus, while 1 case was located $1.5 \mathrm{~cm}$ below the dentate line. Five cases were all flat-uplifted (0-IIa). The average operation time was $28.6 \pm 9.0 \mathrm{~min}$. All 5 patients underwent complete resection, and all of them achieved complete resection. There was no recurrence of the 5 cases for 36-49 months of follow-up.
\end{abstract}

Conclusions: ESD can be used as a method for the treatment of GA-FG.

Keywords: Gastric adenocarcinoma of the fundic gland type; gastric cancer; endoscopic submucosal exfoliation (ESD)

Submitted Mar 15, 2019. Accepted for publication Aug 08, 2019.

doi: $10.21037 /$ tcr.2019.08.19

View this article at: http://dx.doi.org/10.21037/tcr.2019.08.19

\section{Introduction}

Gastric cancer is the second most common malignant tumor worldwide, and the incidence and mortality rate is high $(1,2)$. The most common pathological type of gastric cancer is gastric adenocarcinoma. For gastric adenocarcinoma, the pathological types include the diffuse type, intestinal type, mixed type, and uncertain type $(3,4)$. The intestinal adenocarcinoma type originates from a Helicobacter pylori (HP) infection with atrophic gastric mucosa had been known worldwide (5). However, some traditional intestinaltype gastric cancer was found actually to be gastric type differentiation with different immunohistochemical phenotypes like mucin. Early in 2007, Tsukamoto et al. (6) from Japan initially reported this type of gastric cancer. Then Ueyama et al. (7) reported 10 cases in 2010 and officially named it gastric adenocarcinoma of the fundic gland type (GA-FG). GA-FG originates from the deep part of the non-atrophic normal gastric fundus gland and belongs to a new histological category of gastric cancer. The incidence of GA-FG is unknown, and only dozens of cases have been reported thus far. Furthermore, there is no standard process and method for its treatment.

Endoscopic submucosal exfoliation (ESD) is a standard operation for early gastric cancer which extends as deep as the SM1 and can be larger than $2.0 \mathrm{~cm}$, with some tumors being present in the submucosa (8). It can provide the same 
effect as surgery and provide more accurate pathological evaluation for further treatment. Moreover, the application of ESD in traditional gastric adenocarcinoma can reduce the trauma caused by surgery. Thus, it has become a good choice for the treatment of some precancerous lesions of the stomach (9). However, whether ESD is equally safe and effective in the treatment of GA-FG as it is for traditional gastric adenocarcinoma has rarely been reported on. Therefore, we retrospectively analyzed the data of 5 patients who were definitively diagnosed as GA-FG by postoperative pathology in our hospital. The preoperative preparation and surgical indications of ESD were carried out in accordance with the Japanese Gastric Cancer treatment guidelines 2014 (ver. 4) (10). We analyzed these data to evaluate the value of ESD in GA-FG.

\section{Methods}

\section{Patients}

From Jan 2010 to Dec 2018, a total of 5 patients with GA-FG who was confirmed by postoperative pathology were treated with ESD in the digestive endoscopy center of our hospital. The indications contraindications and perioperative management of ESD were carried out according to the Japanese gastric cancer treatment guidelines 2014 (ver. 4). All cases were found to be suspicious lesions in gastric fundus during ordinary white light endoscopy. Before the operation, the lesions were limited to the mucous layer by endoscopic ultrasonography, and the surface microvessels and microstructures were observed by endoscopic narrow-band imaging (NBI). The 5 patients had already been cleared for coagulation dysfunction, severe cardiopulmonary liver and kidney disease, lymphadenopathy, and other similar conditions that are not compatible with ESD treatment.

Informed consent from all patients and the approval from the Medical Ethics Committee of Affiliated Hospital of Nanjing University of Chinese Medicine (Jiangsu Province Hospital of Chinese Medicine) have been obtained (ID No: 2015NL-094-02).

\section{Operation instruments}

The instruments used in operation included the following: Olympus GIF-Q260J electronic gastric endoscopy (Olympus Co. Ltd., Japan), UM-3R ultrasound probes (frequencies 12 to $20 \mathrm{MHz}$, Olympus Co. Ltd., Japan),
Cook NM-4L-1 injection needle (COOK, Co. Ltd., USA), KD-620LR HOOK knife (Olympus Co. Ltd., Japan), KD-611L IT knife (Olympus Co. Ltd., Japan), FD-1U-1 thermal biopsy (Olympus Co. Ltd., Japan), HX-600-135. Hemostatic clip (Olympus Co. Ltd., Japan), ERBE ICC200HF electro resection device (ERBE Co. Ltd., German), APC300 argon-ion coagulator (ERBE Co. Ltd., German), Transparent cap (Olympus Co. Ltd., Japan), and harmony clip (Micro-tech Co. Ltd., China).

\section{ESD methods}

(I) Mark: the edge of the lesions was determined by white light endoscope assisted with NBI or indigo dyeing, while an argon knife or endoscopic electrosurgical knife were used to mark the lesions. (II) Injection: multi-point injection of saline containing indigo carmine and epinephrine at the lateral submucosa of the marking point. (III) Marginal incision: The endoscopic electric knife was used to cut the mucosa along the lateral side of the marking point. (IV) Dissection: the submucosa was stripped under the lesion by electric knife, and the lesion was resected completely. (V) Wound treatment: for the small blood vessels visible in the wound, APC coagulation or thermal biopsy forceps were used to treat the wound, and if necessary, a harmonious clip was used to close a part of the wound. (VI) Specimen processing: the resected lesions were fixed on the plate with a pin, and the neutral formaldehyde solution was fixed and sent to for pathological examination (10).

\section{Postoperative management and follow-up}

On the first day after ESD, fasting, fluid replacement, a proton pump inhibitor (9), and antibiotics were conventionally used. The quality and quantity of drainage from the gastrointestinal decompression tube, chest pain, abdominal pain, and abdominal sign changes should be focused on. If there were no bleeding or perforation symptoms on the $3 \mathrm{rd}$ day after the operation, the gastrointestinal decompression tube would be removed, and a fluid diet would be taken. If there were symptoms of delayed bleeding such as hematemesis or melena, endoscopy should be performed in time. It is suggested that the endoscopy reexamination should be taken after the operation at 1, 3, 6, and 12 months, and once a year thereafter to see if the patient survives or if the disease recurs. The last follow-up time of these 5 cases was Feb 28, 2019. 


\section{Evaluation indicators and statistical analysis}

The size of the tumor is based on the longest diameter of the tumor provided by the pathology department. Complete resection refers to the whole resection of the lesion, and the perimeter and base of the resected specimen do not include the lesion. If the edge is cauterized, it is considered an incomplete resection (9). Curative resection refers to that pathological diagnosis, which accords with the surgical indication of ESD treatment and has no risk of lymph node metastasis (11). That is to say; traditional surgical treatment does not need to be respected. Intraoperative bleeding is defined as intraoperative bleeding that requires suspension of surgery, replacement of thermal biopsy forceps, etc., which takes a long time and is relatively difficult to deal with, excluding small bleeding or bleeding that can be treated by simple electrocoagulation.

The patient history, lesion size, pathological results, complete resection, curative resection, intraoperative and postoperative complications, and the perioperative management of endoscopic treatment, additional surgery, postoperative radiotherapy, postoperative chemotherapy, etc. were documented and analyzed. SPSS 16.0 software was used for data statistics, and the measurement data are expressed as mean with SD.

\section{Results}

\section{General information}

Among the 5 patients, there were 2 males and 3 females ( $\mathrm{M}: \mathrm{F}=2: 3$ ). The average age was $59.8 \pm 14.4$ years old. Most of them had occasional acid regurgitation and belching discomfort, but these symptoms could be alleviated on their own. Moreover, there was no history of HP infection or digestive tract ulcers. Four patients with recurrent gastric fundus polyps before the operation were treated with APC or EMR.

\section{Preoperative endoscopy performance}

The white light endoscopy showed that 4 cases were located at the fundus, while 1 case was located $1.5 \mathrm{~cm}$ below the dentate line. Five cases were flat-uplifted (0-IIa) mucosal surface flushing, with the surface blood vessel being congested (Figure 1A). The lesion size ranged from 0.8 $1.8 \mathrm{~cm}$ (average, $1.2 \pm 0.4 \mathrm{~cm}$ ). The mucosa did not see signs of Hp infection, and rapid urease method also indicated that
HP infection was negative.

Endoscopic ultrasonography showed a low echo area with a slight thickening of the focal mucosa, and the submucosal and mucosal base layers were intact and continuous. Under the detection of NBI, the boundaries of the 5 patients were clearly visible, the microvascular structure was slightly disordered, and the micro-blood vessels were partially dilated. However, the micro-surface pattern was still regular (Figure 1B, Table 1).

\section{Treatment and patbology}

The average operation time was $28.6 \pm 9.0 \mathrm{~min}$. All 5 patients underwent complete resection, and all achieved complete resection. There was no perforation, and no uncontrolled bleeding or transfer surgery occurred during the endoscopy operation (Figure 1C,D).

Under the microscope, the glandular structures of the lesions were randomly arranged and fused together. They were composed of 2 cells resembling the fundus glandular cells and the fundus gland cells. The irregular glands are invasively growing in the lamina propria or invading 0.2 $0.5 \mathrm{~mm}$ into the submucosa (average $0.36 \pm 0.18 \mathrm{~mm}$ ). The lamina propria and the submucosal invasions were welldifferentiated, mild dysplasia, with no interstitial fibrous connective tissue hyperplasia, no involvement of the vasculature, and mild gastric non-atrophic gastritis in the surrounding gastric mucosa. Atypical glands in the lamina propria and infiltrating submucosa showed the following: MUC6 (+), pepsinogen-I (+), and $\mathrm{H}+/ \mathrm{K}+-$ ATPase (+). The pepsinogen-I positive cells were significantly more numerous than the $\mathrm{H}+\mathrm{K}+-\mathrm{ATP}$ ase positive cells, while MUC2, MUC5AC, and HER-2 were all negative. The proliferation index for Ki-67 which could be an indicator of tumor differentiation was $5 \% \sim 10 \%$. The lesion surface was pitted with epithelial MUC5AC (+) (Figure 2A,B,C,D,E,F, Table 2).

\section{Postoperative follow-up results}

All patients had no delayed bleeding, perforation, stenosis, or other serious complications. One patient whose lesion underwent submucosal infiltration at $500 \mathrm{~nm}$ had proximal gastrectomy one week after the operation. No residual cancer or lymph node metastases were observed from the excised stomach. The other 4 patients had no additional surgery, postoperative radiotherapy, or chemotherapy. All patients 

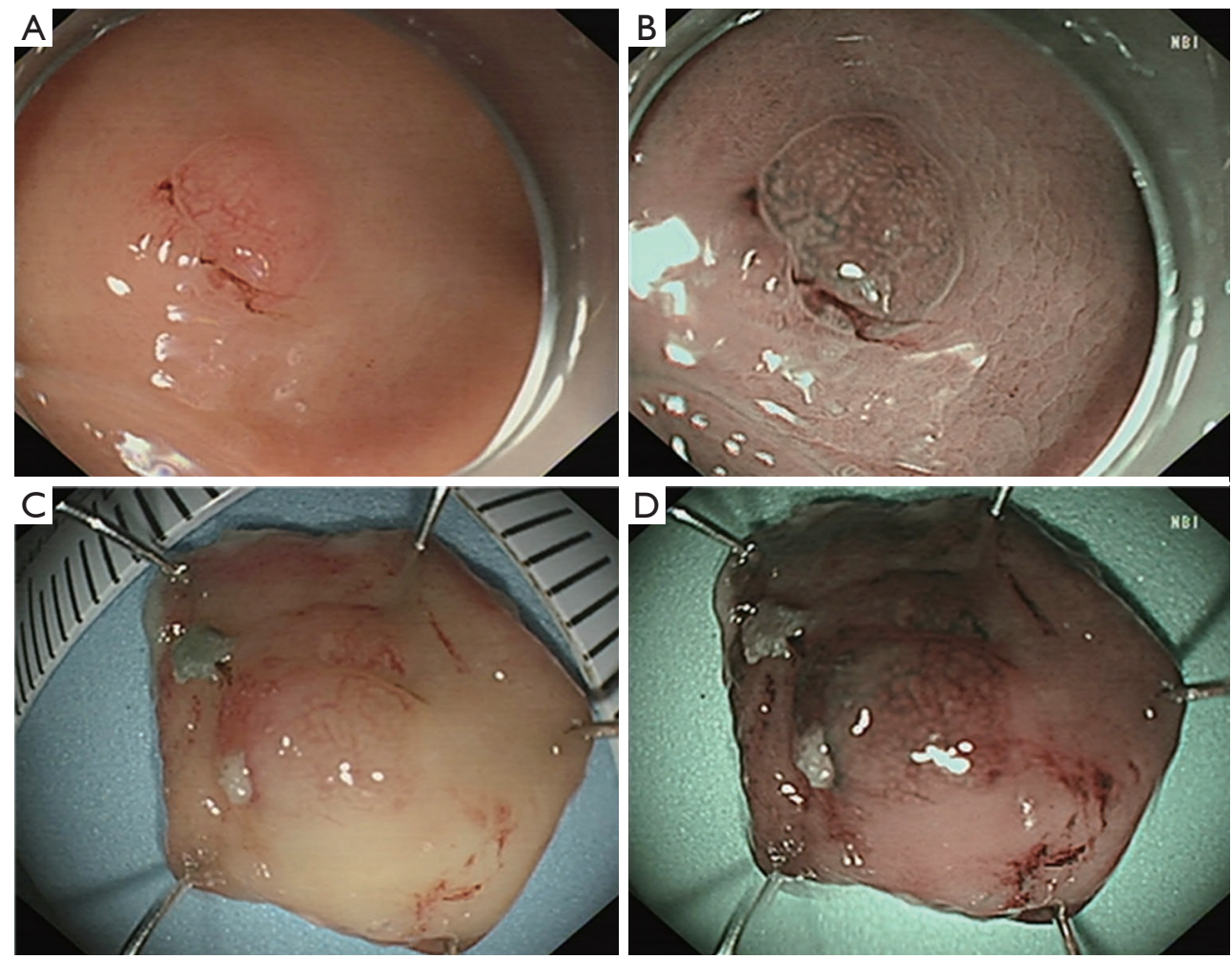

Figure 1 Endoscopic performance of GA-FG. (A) Endoscopic white light: hypothalamic hyperplasia at the fundus, small blood vessels congested, no change in the surrounding mucosa; (B) NBI: the boundary of the lesion is visible, the local micro vessels are slightly expanded, and the microstructure is still regular; (C) white light endoscopic observation of lesions in vitro; (D) NBI observation of lesions in vitro. GAFG, gastric adenocarcinoma of fundic gland type.

Table 1 General characteristics of patients

\begin{tabular}{cccccccccc}
\hline No. & Sex & Age & Operation time & Diameter & Mucosal color & EUS & IMVP & IMSP & Additional surgery \\
\hline 1 & F & 46 & 21 & 0.8 & Flushing & Mucosal thickening & - & - & Yes \\
2 & M & 77 & 30 & 1.5 & Flushing & Mucosal thickening & -+ & -+ & No \\
3 & M & 63 & 42 & 1.8 & Flushing & Mucosal thickening & - & - & No \\
4 & F & 44 & 30 & 1.2 & Fade & Mucosal thickening & - & - & No \\
5 & F & 69 & 20 & 0.8 & Fade & Mucosal thickening & - & - & No
\end{tabular}

-+, weakly positive. EUS, endoscopy ultrasound; IMVP, irregular microvascular pattern; IMSP, irregular microsurface pattern.

were followed up without any loss for 36-49 months (the median follow-up time was $40.4 \pm 5.2$ months). The last telephone follow-up time was February 28, 2019. None of the 5 patients indicated any discomfort in the private prosecution. Endoscopic follow-up was performed at 3, 6, 12, 24, and 36 months after endoscopy operation postoperatively. There was no recurrence of the lesion (Table 2).

\section{Discussion}

GA-FG is a new histological type of gastric cancer that was discovered in recent years and named by Ueyama et al. (7) in 2010. It has low malignant and highly differentiated adenocarcinoma with gastric fundus differentiation. The English literature reports only 50 cases (12), most of these 

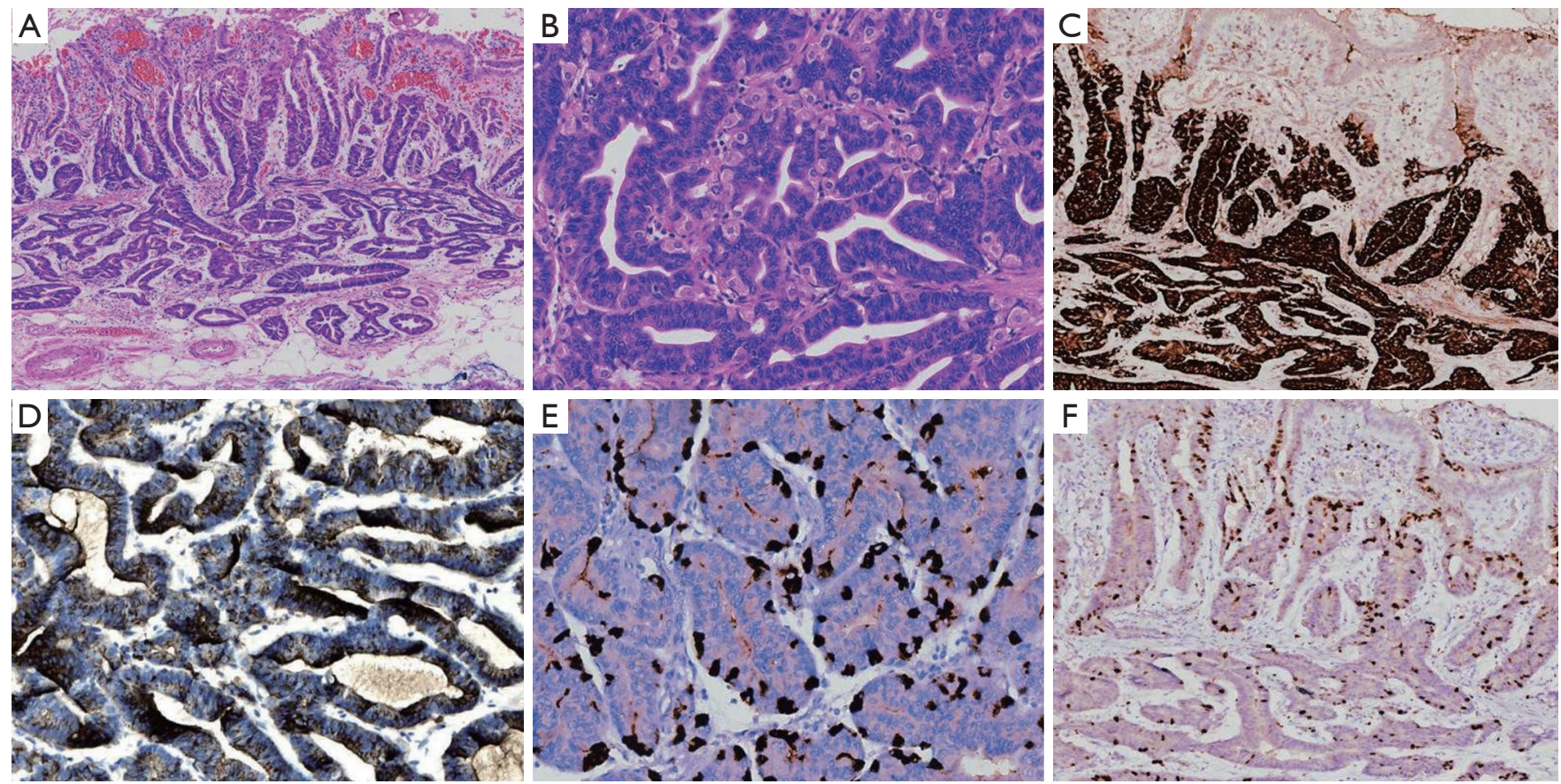

Figure 2 Pathology and immunohistochemistry of GA-FG $(4 \times 10)$. (A) The surface of the tumor is covered with a non-shaped gastric pituitary epithelium. The lamina propria is arranged in a disorderly manner, and the glands are merged with each other and invade the submucosa. (B) The tumor consists of two kinds of cells including the main cells of the fundus gland and the parietal cells. (C) MUC6 (+). (D) pepsinogen-I (+). (E) H+/K+-ATPase (+). (F) Ki-67 has a low proliferation index. GA-FG, gastric adenocarcinoma of fundic gland type.

Table 2 Pathology and follow-up

\begin{tabular}{|c|c|c|c|c|c|c|c|c|}
\hline No. & Depth of lesion & MUC6 & pepsinogen-I & $\mathrm{H}+/ \mathrm{K}+-\mathrm{ATPase}$ & Ki-67 (+) & Follow-up time & Lesion recurrence & $\begin{array}{l}\text { Postoperative } \\
\text { complications }\end{array}$ \\
\hline 1 & SM1 & + & + & + & 3 & 49 & No & GERD \\
\hline 3 & SM1 & + & + & + & 6 & 37 & No & No \\
\hline 4 & SM1 & + & + & + & 8 & 41 & No & No \\
\hline
\end{tabular}

GERD, gastroesophageal reflux disease.

written by Japanese scholars. According to the literature, GA-FG commonly occurred in males (male-female ratio: 1.4:1), and the average age of onset was 42 to 82 years old (5). Most GA-FG patients showed no clinical symptoms or only had the symptoms of gastroesophageal reflux, which was possibly associated with the acid secretion of the undiluted gastric mucosa stomach (13). HP infection was negative in the nearly 50 reported patients. However, about $75 \%$ of the cases had a history of HP infection and eradication (14).
More than $80 \%$ of GA-FG cases were located in the upper third of the stomach, and the rest were located in the middle of the stomach. The lesions were single, small, with an average of $7.5 \mathrm{~mm}$, and $80 \%$ of the cases are $<1 \mathrm{~cm}$. About 3/4 cases were superficial uplift (0-IIa), and the rest were superficially flat $(0-\mathrm{IIb})$ or superficial depression $(0$ IIc) (15). White light endoscopy showed the pattern of GAFG was similar to some submucosal tumors (SMT) such as a neuroendocrine tumor. The boundary of the lesion was unclear, and the color of most reported lesions was faded. 
The dilated blood vessels were visible, and the surrounding mucosa did not shrink (16). NBI showed that the boundary of the lesion was not clear, and irregular microvascular phenotype or irregular micro-surface phenotype was mostly negative (17).

GA-FG lesions are small, low-grade malignant tumors, and are considered to be highly differentiated gastric tumors with a good prognosis. However, GA-FG could also progress to gastric tubular adenocarcinoma (18). Most of the GA-FG infiltrated the submucosa to a depth of no more than $500 \mu \mathrm{m}$, but in very few cases, it could exceed $500 \mu \mathrm{m}$ and even reach as deep as 4,000 $\mu \mathrm{m}$ (19). The current main treatment is ESD resection and surgery, but given the paucity of cases and inadequate follow-up, the treatment of GA-FG is not yet clear.

In this study, the general data of the 5 cases were basically consistent with previous literature reports. It is important to emphasize that in this study, 5 patients had undergone gastroscopy at our center every 12 months since 2012, and 4 patients had a history of repeated resection of polyps. The polyp pathology suggested gastric fundus polyps or gastric adenoma. Thus, we hypothesize that gastric fundus glandular polyps and fundic gland adenomas may be precancerous lesions of GA-FG. In this study, 5 patients underwent ESD, and the lesions were completely resected. It is suggested that ESD can completely remove the lesion. There were no serious complications such as massive bleeding and perforation during and after operation; that is, ESD was safe for the treatment of GA-FG.

Our follow-up results showed that the patient undergoing surgical treatment had symptoms of gastroesophageal reflux after surgery and required drug controlling symptoms, while the other 4 patients had no particular discomfort. All patients had no tumor recurrence after 36 months of follow-up. Currently, gastroscopy follow-up is being performed every 12 months. The results of follow-up have demonstrated that ESD treatment of GA-FG did not seem to differ from the same indications, contraindications, and additional surgery for other specialties of gastric cancer for more than 36 months. Because of the rare incidence of this disease, this study also has limitations. Only 5 cases of medical records were included, and the maximum infiltration depth was only $500 \mu \mathrm{m}$ below the submucosa. More reliable results have yet to be further explored by large samples and multiple center studies. Overall, ESD is safe and effective for the treatment of GA-FG and maybe the main method for the treatment of GA-FG.

\section{Acknowledgments}

Funding: This study is supported by the second batch of special research projects for the construction of the National TCM Clinical Research fund (No. JDZX2015086), an innovation fund of the Jiangsu Hospital of Chinese Medicine (grant no. Y2018CX57) and Jiangsu Province "333 Project" Training Fund (BRA2017551).

\section{Footnote}

Conflicts of Interest: All authors have completed the ICMJE uniform disclosure form (available at http://dx.doi. org/10.21037/tcr.2019.08.19). The authors have no conflicts of interest to declare.

Ethical Statement: The authors are accountable for all aspects of the work in ensuring that questions related to the accuracy or integrity of any part of the work are appropriately investigated and resolved. The study was conducted in accordance with the Declaration of Helsinki (as revised in 2013). Informed consent from all patients and the approval from the Medical Ethics Committee of Affiliated Hospital of Nanjing University of Chinese Medicine (Jiangsu Province Hospital of Chinese Medicine) have been obtained (ID No. 2015NL-094-02).

Open Access Statement: This is an Open Access article distributed in accordance with the Creative Commons Attribution-NonCommercial-NoDerivs 4.0 International License (CC BY-NC-ND 4.0), which permits the noncommercial replication and distribution of the article with the strict proviso that no changes or edits are made and the original work is properly cited (including links to both the formal publication through the relevant DOI and the license). See: https://creativecommons.org/licenses/by-nc-nd/4.0/.

\section{References}

1. Chen $\mathrm{W}$, Zheng R, Baade PD, et al. Cancer statistics in China, 2015. CA Cancer J Clin 2016;66:115-32.

2. Siegel RL, Miller KD, Jemal A. Cancer statistics, 2019. CA Cancer J Clin 2019;69:7-34.

3. Ackerman LV, Rosai J. The pathology of tumors. 4. Grading, staging and classification of neoplasms. CA Cancer J Clin 1971;21:368-78.

4. Pyo JH, Lee H, Min BH, et al. Early gastric cancer with 
a mixed-type Lauren classification is more aggressive and exhibits greater lymph node metastasis. J Gastroenterol 2017;52:594-601.

5. Yamamoto Y, Fujisaki J, Omae M, et al. Helicobacter pylori-negative gastric cancer: Characteristics and endoscopic findings. Dig Endosc 2015;27:551-61.

6. Tsukamoto T, Yokoi T, Maruta S, et al. Gastric adenocarcinoma with chief cell differentiation. Pathol Int 2007;57:517-22.

7. Ueyama H, Yao T, Nakashima Y, et al. Gastric adenocarcinoma of fundic gland type (chief cell predominant type): proposal for a new entity of gastric adenocarcinoma. Am J Surg Pathol 2010;34:609-19.

8. Kim KH, Kim CG, Kim YW, et al. Phlegmonous Gastritis with Early Gastric Cancer. J Gastric Cancer 2016;16:195-9.

9. Jung YD, Chung WC, Kim YJ, et al. Gastric neuroendocrine tumor mimicking early gastric cancer. Gastrointest Endosc 2017;86:741-2.

10. Japanese Gastric Cancer Association. Japanese gastric cancer treatment guidelines 2014 (ver. 4). Gastric Cancer 2017;20:1-19.

11. Chiu PWY, Uedo N, Singh R, et al. An Asian consensus on standards of diagnostic upper endoscopy for neoplasia. Gut 2019;68:186-97.

12. Benedict MA, Lauwers GY, Jain D. Gastric Adenocarcinoma of the Fundic Gland Type: Update and Literature Review. Am J Clin Pathol 2018;149:461-73.

Cite this article as: Li Y, Lu Y, Yu C, Xiao J, Han S. Efficacy analysis of endoscopic submucosal dissection in gastric adenocarcinoma of fundic gland type: five cases of more than 36 months of follow-up. Transl Cancer Res 2019;8(5):17341740. doi: $10.21037 /$ tcr.2019.08.19
13. Tajima Y, Murakami T, Saito T, et al. Distinct Involvement of the Sonic Hedgehog Signaling Pathway in Gastric Adenocarcinoma of Fundic Gland Type and Conventional Gastric Adenocarcinoma. Digestion 2017;96:81-91.

14. Imagawa A, Sano N. Gastric adenocarcinoma of the fundic gland (chief cell predominant type) with brownish pigmentation. Gastrointest Endosc 2018;87:1358-9.

15. Kai K, Satake M, Tokunaga O. Gastric adenocarcinoma of fundic gland type with signet-ring cell carcinoma component: A case report and review of the literature. World J Gastroenterol 2018;24:2915-20.

16. Chiba T, Kato K, Masuda T, et al. Clinicopathological features of gastric adenocarcinoma of the fundic gland (chief cell predominant type) by retrospective and prospective analyses of endoscopic findings. Dig Endosc 2016;84:722-30.

17. Takeda S, Mitoro A, Namisaki T, et al. Gastric adenocarcinoma of fundic gland type (chief cell predominant type) with unique endoscopic appearance curatively treated by endoscopic submucosal resection. Acta Gastroenterol Belg 2015;78:340-3.

18. Kino H, Nakano M, Kanamori A, et al. Gastric Adenocarcinoma of the Fundic Gland Type after Endoscopic Therapy for Metachronous Gastric Cancer. Intern Med 2018;57:795-800.

19. Ueyama H, Matsumoto K, Nagahara A, et al. Gastric adenocarcinoma of the fundic gland type (chief cell predominant type). Endoscopy 2014;46:153-7. 